\title{
Terahertz quantum cascade lasers in a magnetic field
}

\section{Citation}

Tamosiunas, V., R. Zobl, J. Ulrich, K. Unterrainer, R. Colombelli, C. Gmachl, K. West, L. Pfeiffer, and F. Capasso. 2003. "Terahertz Quantum Cascade Lasers in a Magnetic Field." Applied Physics Letters 83 (19): 3873-75. https://doi.org/10.1063/1.1626018.

\section{Permanent link}

http://nrs.harvard.edu/urn-3:HUL.InstRepos:41371480

\section{Terms of Use}

This article was downloaded from Harvard University's DASH repository, and is made available under the terms and conditions applicable to Other Posted Material, as set forth at http:// nrs.harvard.edu/urn-3:HUL.InstRepos:dash.current.terms-of-use\#LAA

\section{Share Your Story}

The Harvard community has made this article openly available.

Please share how this access benefits you. Submit a story.

Accessibility 


\title{
Terahertz quantum cascade lasers in a magnetic field
}

\author{
V. Tamosiunas, R. Zobl, J. Ulrich, and K. Unterrainera) \\ Institut für Festkörperelektronik, Technische Universität Wien, A-1040 Wien, Austria \\ R. Colombelli, ${ }^{\text {b) }}$ C. Gmachl, K. West, and L. Pfeiffer \\ Bell-Laboratories, Lucent Technologies, 600 Mountain Avenue, Murray Hill, New Jersey 07974 \\ F. Capasso \\ Harvard University, Division of Engineering and Applied Sciences, Cambridge, Massachusetts 02138
}

(Received 31 March 2003; accepted 17 September 2003)

\begin{abstract}
We have investigated the behavior of a terahertz quantum cascade laser in an external magnetic field. A reduction of the threshold current density and a simultaneous enhancement of the laser emission intensity are observed. Although several mechanisms can induce this effect, the suppression of nonradiative Auger-intersubband transitions through Landau quantization of the in-plane electron motion is the most probable candidate. In addition, the injection rate via resonant inter-Landau-level transfer and the waveguide properties are modulated by the field. We also observed clear shifts of the emission spectra when the external magnetic field is applied, while operating the device at constant voltage or current. (C) 2003 American Institute of Physics.
\end{abstract}

[DOI: $10.1063 / 1.1626018]$

A quantum cascade laser (QCL) operating in the terahertz $(\mathrm{THz})$ range of the electromagnetic spectrum was recently demonstrated by Köhler et al. ${ }^{1}$ Continuous wave operation was reported as well, ${ }^{2}$ but the further improvement of the THz QCLs is a challenge for several reasons related to the intersubband population dynamics and to the waveguide properties. Electron-electron and interface roughness scattering represent the main scattering mechanisms at low temperatures in this range of intersubband energies. They lead to fast carrier relaxation and they counteract population inversion.

Blank and Feng ${ }^{3}$ proposed to increase the intersubband lifetime by applying a magnetic field normal to the epitaxial layers. The additional quantization (induced by the magnetic field) of the in-plane electronic motion dramatically modifies the electron-electron scattering in the Landau-quantized energy spectrum. As a matter of fact, the recently observed enhancement of the luminescence intensity of GaAs/AlGaAs ${ }^{4}$ and InGaAs/InAlAs ${ }^{5} \mathrm{THz}$ quantum cascade structures originates from this modification. Landau quantization was also exploited to control electron-LO phonon scattering in midinfrared lasers. ${ }^{6}$

In this work, we studied the behavior of a $4.5 \mathrm{THz}$ QCL in an external magnetic field. The structure consists of 100 periods of a layer system of GaAs wells and $\mathrm{Al}_{0.15} \mathrm{Ga}_{0.85} \mathrm{As}$ barriers (as proposed in Ref. 1) sandwiched between two $n$ + -doped contact layers, and it was grown on a semi insulating GaAs substrate using molecular beam epitaxy. The bottom layer is $500 \mathrm{~nm}$ thick and it is doped to $n=4$ $\times 10^{18} \mathrm{~cm}^{-3}$, while the top layer is $100 \mathrm{~nm}$ thick and doped to $n=7 \times 10^{18} \mathrm{~cm}^{-3}$.

The laser devices were processed into ridges by reactive ion etching through the active zone down to the bottom con-

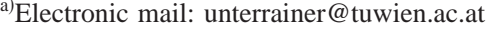

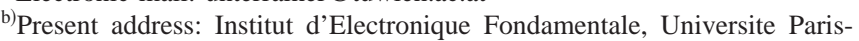
Sud, 91405 Orsay, France.
}

tact layer. Metallic contacts ( $\mathrm{Ge} / \mathrm{Au} / \mathrm{Ni} / \mathrm{Au}, 15 / 30 / 14 / 200$ $\mathrm{nm}$ ) were evaporated onto the bottom contact layer, and as two $10 \mu \mathrm{m}$ wide contact stripes on top of the ridge. The sample was then annealed for $1 \mathrm{~min}$ at a temperature of $430^{\circ} \mathrm{C}$, and a surface-plasmon carrying Ti/Au layer $(10 / 400$ $\mathrm{nm}$ ) was sputtered on top of the ridge to complete the top contact and to provide waveguiding.

The magnetic field measurements were performed with the device mounted in a cryostat equipped with two superconducting magnets. Several different detectors can be used in conjunction with this system, namely a magnetic field tunable InSb cyclotron resonance detector, a broadband $\mathrm{Ga}$ doped Ge detector, or a Si bolometer. The last detector was used with an external Fourier-transform infrared spectrometer (FTIR): it has therefore been possible to record highresolution spectra of the THz QCL under the application of the magnetic field.

The response of the InSb detector to our $\mathrm{THz}-\mathrm{QCL}$ emission is shown in Fig. 1(a) (inset) and it displays two peaks: one originates from impurity bound and the other one from free carrier transitions in the detector. The spectral response of the detector was tuned by scanning the detector magnetic field. ${ }^{4}$ Each of the two peaks has a full width at half maximum of approximately $0.6 \mathrm{meV}$. Although individual laser lines cannot be resolved with the InSb detector, the average frequency shift of the entire laser spectra can be detected.

A $100 \mu \mathrm{m}$ wide device was selected for the magnetic field measurements because it exhibited lasing for a very broad current range. A broadband Ge detector was used to measure the light versus current characteristic of the device for several values of the magnetic field applied to the sample [see Fig. 1(a)]. We observed a clear reduction of the threshold current density with increasing applied fields. For example, the threshold is $1.4 \mathrm{~A}$ at $0 \mathrm{~T}$, while it decreases to 0.52 $\mathrm{A}$ at 4.2 $\mathrm{T}$. The laser emission intensity increases substan- 

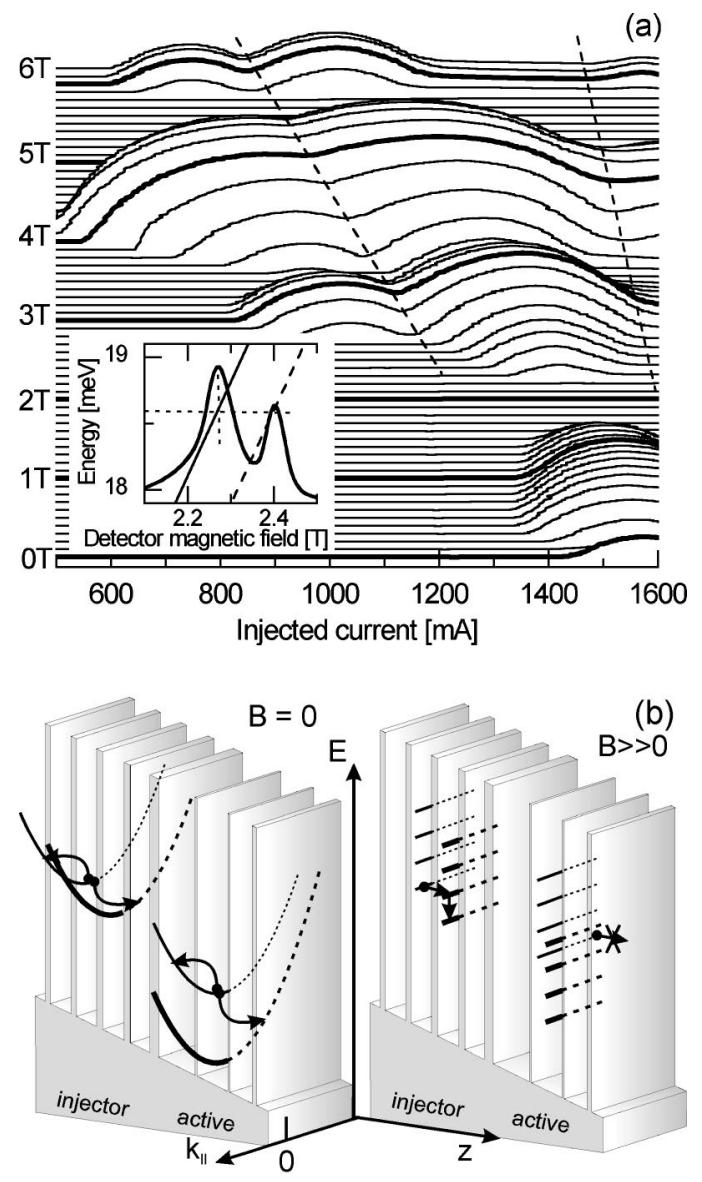

FIG. 1. (a) Laser emission intensity as a function of the injected current at different magnetic fields applied to the sample. The pulse width was $150 \mathrm{~ns}$ at $90 \mathrm{kHz}$ repetition rate. [(a) inset] Response of the InSb detector vs applied detector magnetic field when illuminated by the THz QCL. The solid calibration line corresponds to the free electron cyclotron resonance, the dashed line to the impurity bound resonance. (b) Energy levels in the injector and in the active region without and with strong applied magnetic field. The parabolic in-plane dispersion of the subbands allows electron-electron scattering and nonradiative recombination both in the injector and in the active region. A magnetic field breaks the subbands into ladders of Landau levels. For certain values of the magnetic field it is possible to achieve favorable conditions for fast energy relaxation in the injector (indicated by straight arrows), and to suppress nonradiative relaxation in the active region (crossed arrow).

tially for certain values of the magnetic field when compared to $B=0 \mathrm{~T}$. Furthermore, we observe oscillations of the intensity as a function of the current, and with increasing magnetic fields we observe oscillations with a larger period.

Let us first discuss the dependence of the laser output power as a function of the magnetic field. The application of a magnetic field leads to an increase of the laser intensity of a factor of four at $B=4.2 \mathrm{~T}$ [see Fig. 1(a)], an effect that is induced by the discretization of the energy spectrum. In the absence of a magnetic field, the energy conservation condition for intersubband Auger scattering processes is satisfied by a continuum of energy changes $\Delta E$. However, under the application of a magnetic field the only allowed energy changes $\Delta E$ are multiples of the cyclotron resonance energy $\hbar \omega_{c}$. Since the Auger scattering rate is inversely proportional to the exchanged energy and momentum, the Landaulevel splitting induced by the magnetic field reduces the nonradiative relaxation rate, thereby increasing the laser efficiency. This is about two times larger than the spacing between ad-
efficiency.
Downloaded 21 May 2008 to 128.103.60.225. Redistribution subject to AlP license or copyright; see http://apl.aip.org/apl/copyright.jsp
We will now discuss in detail how the magnetic field affects the laser emission. The laser output power is proportional to the population inversion which, in turn, is directly proportional to the injection rate and inversely proportional to the nonradiative relaxation rate. If we assume that the injection rate is not influenced by the magnetic field, we can expect an oscillatory increase of the laser emission. The minima of the laser output power should correspond to magnetic field values that meet the following resonance condition:

$$
E_{2}-E_{1}=\frac{\hbar e B_{N}}{m^{*}} N
$$

Here $E_{2}-E_{1}$ is the energy difference between the lowest Landau levels of the upper and lower laser transition, $\hbar$ is the Planck constant, $e$ is the elementary charge, $B_{N}$ is magnetic flux density, $m^{*}$ is effective mass, and $N$ is an integer. The resonant tunneling between Landau levels opens up an additional nonradiative relaxation channel via the Landau ladder $(|1, \mathrm{~N}\rangle,|1, \mathrm{~N}-1\rangle, \ldots|1,0\rangle)$. The consequent reduction of population inversion induces a decrease of the laser emission intensity. It should be pointed out that a theoretical model proposed by Raikh and Shahbazyan ${ }^{7}$ well describes the momentum transfer caused by interface roughness or impurities, a mechanism which is necessary for resonant transfer between Landau levels of different subbands. Figure 1(a) clearly shows that the laser stops working at these magnetointersubband resonances. We observe the $N=4,3$, and 2 resonances at 2.6, 3.5, and 5.4 T. As expected, the laser intensity reaches its maximum between the $N=3$ and $N=2$ resonance.

So far we have assumed that the injection rate is not affected by the magnetic field. This assumption is not entirely justified since additional features are observed in the magnetic field dependence of the laser intensity. An efficient injector requires high scattering rates in order to achieve good injection into the upper laser state as well as fast extraction out of the lower laser state. Therefore, it would be advantageous to have magneto-intersubband resonances between all the injector states, between the last injector state and the upper laser state, and between the lower laser state and the first extractor state. The energy separation between the injector states is small $(\sim 3.1-3.8 \mathrm{meV})^{8}$ and the condition for complete magnetic quantization

$$
\frac{\hbar e B}{m^{*}}>\Delta E_{\text {injector }}
$$

is reached at relatively low magnetic fields. The optimum performance of the laser should be achieved for $\left(\hbar e B / m^{*}\right) M=\Delta E_{\text {injector }}$ and simultaneously $\left(\hbar e B / m^{*}\right) N$ $\neq \Delta E_{\text {laser }}$, where $N$ and $M$ are integers [Fig. 1(b)]. The interplay between increased injector efficiency and reduced nonradiative relaxation rates determines the detailed dependence of the laser intensity on the magnetic field. Due to the complex structure of the injector a quantitative model is difficult to obtain. However, it is obvious that at $4.2 \mathrm{~T}$ optimum performance is achieved. This corresponds to $N=2.5$. If we assume $M=1$ (i.e., the first magneto-intersubband resonance for the injector) we obtain an injector spacing of $7.4 \mathrm{meV}$. 


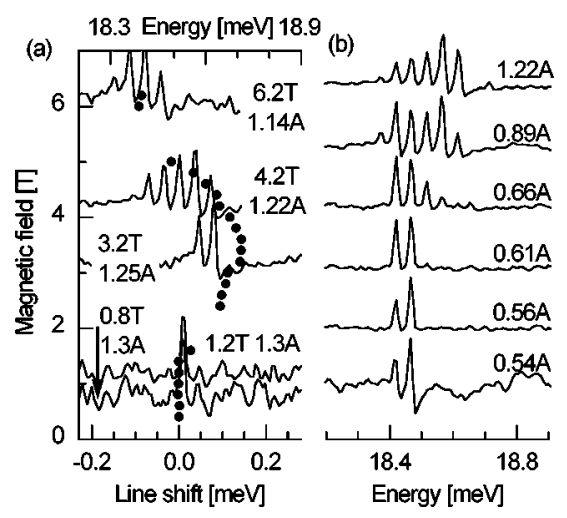

FIG. 2. (a) Frequency of the emission line vs magnetic field applied to the sample, measured by the InSb detector (dots), and high-resolution spectra measured by the external FTIR for several different magnetic fields (lines). The pulser settings were held constant in both measurements to allow a direct comparison. However, currents or voltages were not exactly constant due to the impedance change of the THz QCL. (b) High-resolution spectra for different injected currents at a fixed magnetic field of $4.2 \mathrm{~T}$. Multimode emission with a spacing of about $0.05 \mathrm{meV}$ and a Stark shift are observed.

jacent injector states without a magnetic field. However, the oscillations of the emission intensity with injector current [dashed line in Fig. 1(a)] suggest that in the injector there is an energy spacing comparable to the cyclotron resonance energy. These intensity oscillations occur only for magnetic fields higher than $3 \mathrm{~T}$, where the cyclotron resonance energy exceeds the design injector energy spacing. At $4.2 \mathrm{~T}$ we observe two oscillations when the current is increased from 0.9 to $1.5 \mathrm{~A}$, while the emission wavelength tunes less than 0.5 meV only.

In addition to the increased intensity we observe a shift of the laser wavelength in the magnetic field. A clear blue shift of $0.15 \mathrm{meV}$ is observed for magnetic fields between 0 and 3.2 T [Fig. 2(a)], while for higher magnetic fields we observe a redshift of the emission. To rule out simple bandstructure effects due to changes of the electric field in the active region, we have performed additional measurements at constant bias and current (see Fig. 3). This has been possible due to the very wide operating range of the laser. An additional higher energy emission line was consistently observed at 3.2 and $4.2 \mathrm{~T}$ at constant voltage conditions [Fig. $3(\mathrm{a})$. Constant current and constant voltage spectra at $4.2 \mathrm{~T}$ display the strongest emission line at $18.6 \mathrm{meV}$. This same emission line is instead absent at $6.2 \mathrm{~T}$ [Fig. 3(b)], and the redshift exceeds the mode spacing of the resonator by approximately five times. We can therefore safely exclude band structure effects as a possible cause for the shift.

Changes in the level population remain as a possible cause for the frequency shift of the emission spectra. A combination of Bloch gain, conventional intersubband gain, and many body effects like the depolarization effect can cause these emission line shifts.

In a recent work, Willenberg et al. ${ }^{9}$ showed that the gain in QCLs can indeed be modeled as a superposition of intersubband gain (that needs population inversion), and of Bloch gain (which instead does not require inversion). ${ }^{9}$ The latter exhibits an asymmetric profile with gain below the intersubband resonance and absorption above it. The depolarization effect vanishes for equal populations of the laser levels,

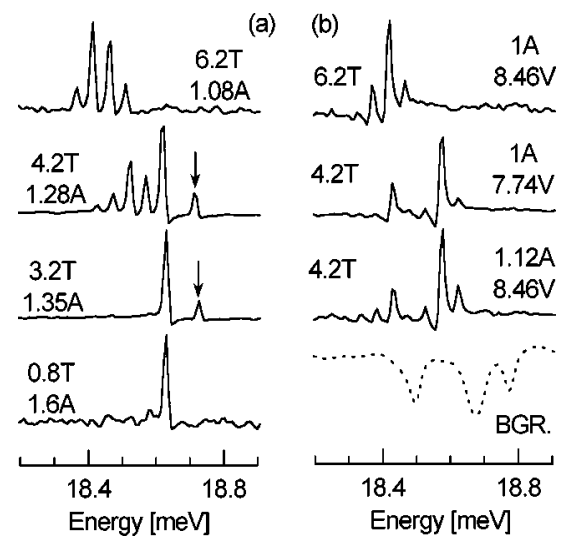

FIG. 3. (a) Constant bias voltage measurement: The measured voltage was set to $9.5 \mathrm{~V}$ for all measurements at a pulse length of $200 \mathrm{~ns}$ and at a repetition rate of $94 \mathrm{kHz}$. An additional emission line appears consistently above $18.7 \mathrm{meV}$ at 3.2 and $4.2 \mathrm{~T}$ (indicated by an arrow). The $18.65 \mathrm{meV}$ emission line is not recorded due to a water absorption line. (b) Long pulse (400 ns) measurements at $6.2 \mathrm{~T}(1 \mathrm{~A}$ and $8.46 \mathrm{~V}$, as measured on the sample), and for $4.2 \mathrm{~T}$ at constant voltage and current. A voltage change of $0.7 \mathrm{~V}$ had a much smaller effect than the magnetic field change. A background spectrum with water vapor absorption lines is also presented (BGR).

while a redshift of the transition energy should instead be observed when the population inversion increases. ${ }^{10}$ In addition, a larger population inversion should lead to an increase of the emission intensity. We observed a very clear intensity maximum at $4.2 \mathrm{~T}$ together with an emission wavelength redshift. An even larger redshift was observed at $6.2 \mathrm{~T}$, but the maximum emission intensity was reduced. The emission intensity decrease can be explained by a reduction of the confinement factor at high magnetic fields, due to a substantial change of the refractive index of the bottom $n^{+}$layer. Our numerical simulation of the waveguide properties in a magnetic field confirms the possibility of such reduction. However, more detailed theoretical and experimental studies are needed to quantitatively calculate the contribution of the Bloch gain and of the many body effects to the spectral properties of $\mathrm{THz}$ QCLs. Since the population inversion in these devices is still severely limited at higher temperatures, a more detailed understanding of the collective nature of the intersubband or Bloch gain is needed. This has to be done not only theoretically, but also by studying the line shifts as a function of carrier (doping) concentration.

The authors acknowledge support by the Austrian Science Foundation (START, ADLIS, SFB) and the European Community IST program (Teravision, Supersmile).

${ }^{1}$ R. Köhler, A. Tredicucci, F. Beltram, H. E. Beere, E. H. Linfield, A. G. Davies, D. A. Ritchie, R. C. Iotti, and F. Rossi, Nature (London) 417, 156 (2002).

${ }^{2}$ L. Ajili, G. Scalari, D. Hofstetter, M. Beck, J. Faist, H. Beere, G. Davis, E. Linfield, and D. Ritchie, Electron. Lett. 38, 1675 (2002).

${ }^{3}$ A. Blank and S. Feng, J. Appl. Phys. 74, 4795 (1993).

${ }^{4}$ J. Ulrich, R. Zobl, K. Unterrainer, G. Strasser, and E. Gornik, Appl. Phys. Lett. 76, 19 (2000).

${ }^{5}$ S. Blaser, M. Rochat, M. Beck, D. Hofstetter, and J. Faist, Appl. Phys. Lett. 81, 67 (2002).

${ }^{6}$ C. Becker, C. Sirtori, O. Drachenko, V. Rylkov, D. Smirnov, and J. Leotin, Appl. Phys. Lett. 81, 2941 (2002).

${ }^{7}$ M. E. Raikh and T. V. Shahbazyan, Phys. Rev. B 49, 5531 (1994).

${ }^{8}$ R. Köhler, R. C. Iotti, A. Tredicucci, and F. Rossi, Appl. Phys. Lett. 79, 3920 (2001)

${ }^{9}$ H. Willenberg, G. H. Döhler, and J. Faist, Phys. Rev. B 67, 085315 (2003).

${ }^{10}$ H. C. Liu and A. J. SpringThorpe, Phys. Rev. B 61, 15629 (2000). 